Original Article

\title{
Improvements in tissue blood flow and lumbopelvic stability after lumbopelvic core stabilization training in patients with chronic non-specific low back pain
}

\author{
Aatit Paungmali ${ }^{1)^{*}}$, Leonard Joseph Henry ${ }^{1,2)}$, Patraporn Sitilertpisan ${ }^{1)}$, Ubon Pirunsan ${ }^{1)}$, \\ SuREePorn UthaikHupl) \\ 1) Department of Physical Therapy, Faculty of Associated Medical Sciences, Chiang Mai University: \\ Chiang Mai 50200, Thailand \\ 2) Physiotherapy Program, School of Rehabilitation Sciences, Faculty of Health Sciences, University \\ Kebangsaan Malaysia, Malaysia
}

\begin{abstract}
Purpose] This study investigated the effects of lumbopelvic stabilization training on tissue blood flow changes in the lumbopelvic region and lumbopelvic stability compared to placebo treatment and controlled intervention among patients with chronic non-specific low back pain. [Subjects and Methods] A total of 25 participants ( 7 males, 18 females; mean age, $33.3 \pm 14.4$ years) participated in this within-subject, repeated-measures, doubleblind, placebo-controlled comparison trial. The participants randomly underwent three types of interventions that included lumbopelvic stabilization training, placebo treatment, and controlled intervention with 48 hours between sessions. Lumbopelvic stability and tissue blood flow were measured using a pressure biofeedback device and a laser Doppler flow meter before and after the interventions. [Results] The repeated-measures analysis of variance results demonstrated a significant increase in tissue blood flow over the lumbopelvic region tissues for post- versus pre-lumbopelvic stabilization training and compared to placebo and control interventions. A significant increase in lumbopelvic stability before and after lumbopelvic stabilization training was noted, as well as upon comparison to placebo and control interventions. [Conclusion] The current study supports an increase in tissue blood flow in the lumbopelvic region and improved lumbopelvic stability after core training among patients with chronic nonspecific low back pain.

Key words: Low back pain, Tissue blood flow, Lumbopelvic stability training
\end{abstract}

(This article was submitted Oct. 8, 2015, and was accepted Nov. 19, 2015)

\section{INTRODUCTION}

Chronic low back pain is an increasing health problem that leads to lost work time and income ${ }^{1,2)}$. Core stabilization of the lumbar spine has been highlighted as one of the key factors that provides spinal stability and prevents lumbopelvic region injury ${ }^{1,3,4)}$. Increasing evidence suggests that lumbopelvic core stabilization training (LPST) provides beneficial effects in the treatment of low back pain ${ }^{2,5-7)}$. In contrast, many studies have argued these potential findings ${ }^{8,9)}$. An important clinical question arises in practice about whether the pain relief effects from LPST are due to improved blood flow to the lumbopelvic region or improved lumbopelvic stability. Although the mechanical effects of LPST on the core stabilizing muscles have been proven, the circulatory effects of LPST in the lumbopelvic region have not been well established. A well-controlled design is needed to prove the clinical benefits of core stabilization exercises in terms of circulatory effects and lumbopelvic stability.

*Corresponding author. Aatit Paungmali (E-mail: aatit.p@cmu.ac.th)

(C2016 The Society of Physical Therapy Science. Published by IPEC Inc.

This is an open-access article distributed under the terms of the Creative Commons Attribution Non-Commercial No Derivatives (by-nc-nd) License $<$ http://creativecommons.org/licenses/by-nc-nd/4.0/>. 
Any knowledge of the circulatory effects and mechanical effects of LPST will enable researchers and clinicians to understand their clinical benefits and apply LPST in patients with chronic low back pain.

Considerable attention has been given to the various aspects of LPST and lumbopelvic stability. Evidence has shown the importance of lumbopelvic core stability among patients with low back pain. The timely activation of lumbopelvic core stability is reportedly necessary to proper lumbopelvic region function ${ }^{13}, 14$ ). Many studies have proven that the core muscles including the transversus abdominis, multifidus, and lower fibers of the internal oblique contract effectively on time prior to activation of the trunk movements that cause a protective feed-forward mechanism in the spine ${ }^{16-18)}$. Some other studies pointed out that the motor control of the lumbar region contributes to compression of the pelvic ring through the activation of force closure muscles ${ }^{15,16}$. Several other studies supported the hypotheses that aberrant lumbopelvic stability (LPS) remains a main cause of ongoing pain in chronic lumbopelvic presentations ${ }^{18,19)}$. In such cases, LPST reportedly produced therapeutic benefits to patients by relieving pain and improving motor control ${ }^{5,7)}$. While the theoretical concepts such as motor control strategies, core muscles contractility, and therapeutic benefits of LPST are well explained, a paucity of knowledge exists in the literature over the claimed circulatory effects such as LPST-induced tissue blood flow (TBF) changes in patients with chronic low back pain.

In clinical reasoning, the circulatory effects and related mechanical effects of LPST become topics of interest when chronic non-specific low back pain improves after LPST. First, it is important to reflect on the potential mechanism behind the circulatory effects that add therapeutic claims to LPST. Second, it is necessary to quantify the LPS if the motor control improves due to LPST. The vicious cycle theory proposes that muscle activity-induced ischemia from vascular compromise causes the accumulation of pain metabolites and becomes a source of further pain ${ }^{20)}$. Therefore, it is possible that the same phenomenon of the vicious cycle theory may occur in patients with chronic non-specific low back pain and lead to vascularity impairments of the lumbopelvic sources that may further lead to inflammatory metabolite accumulation and ongoing nociception. TBF is an element that is indicative of tissue health and the potential for tissue healing after injury ${ }^{21)}$. The blood flow to the tissues is also advocated as a measure of the physiological effects of therapeutic treatments ${ }^{21)}$. A lack of TBF may induce acidity in the working muscle, which can cause further pain ${ }^{25)}$. In this context, it may be appropriate to monitor the TBF changes on the lumbopelvic muscle along with lumbopelvic region stability changes after LPST.

To our knowledge, a collective understanding on the circulatory effect and mechanical effect of LPST among patients with chronic non-specific low back pain has not been established to date. Therefore, the main aim of this study was to investigate the effects of LPST on TBF changes and LPS among patients with chronic non-specific low back pain. The current study hypothesized that LPST might significantly improve TBF and LPS compared to placebo exercise intervention using a passive automated cycling and resting condition as a controlled intervention. The findings of such a study might enhance the general clinical understanding of the circulatory-mechanical effects of LPST in patients with chronic non-specific low back pain.

\section{SUBJECTS AND METHODS}

A total of 25 participants ( 7 males, 18 females; mean age, $33.33 \pm 14.37$ years) with chronic non-specific low back pain voluntarily participated in this within-subject, repeated-measures, double-blind, and placebo-controlled comparison trial. All of the participants were recruited from the community and university area using pre-defined inclusion and exclusion criteria, while the study was conducted in an outpatient physiotherapy department of a university teaching hospital. The inclusion criteria included: age 20-55 years old, mild to moderate back pain, visual analog scale pain score of 2-7 of 10, pain present for $>3$ months in the area between the 12th rib to the gluteal folds. The exclusion criteria were as follows: referred pain or neurological involvement in the lower limbs, history of past surgery, and history of injury in the previous 3 months. All of the participants were instructed not to take stimulants, medications, or alcohol or participate in heavy physical activities at least 8 hours prior to the test. All participants gave written informed consent to join the study and a university ethical committee approved the study per the standards of the Declaration of Helsinki, Finland.

In terms of interventions, the participants randomly underwent 15 minutes of three different experimental conditions of LPST, placebo (passive cycling), and control (rest) with a 48-hour interval between sessions. All of the experimental sessions were conducted in a controlled environmental room (i.e., temperature of $24.5 \pm 0.5^{\circ} \mathrm{C}$, relative humidity of $60 \pm$ $5 \%$ ). All three exercise interventions were administered with the participants in a supine crook-lying position. The LPST was performed on a Pilates Power Gym Transformer (Thane Fitness, UK) per an established training protocol ${ }^{10)}$. An air pressure biofeedback unit was placed beneath the lumbar spine from L2 to S1 and inflated to $40 \mathrm{mmHg}$. A total of seven different core stability training positions were employed in the study to train the participants' core muscles. These seven different positions and movements were as follows: core with alternate hip abduction, core with alternate knee raise, core with both arms adducted, core with both arms extended, core with alternate arm lifts, core with alternate leg lifts, and core with alternate leg and arm lifts. All of the movements were performed using core muscle contraction through abdominal hollowing and co-contraction of the trunk muscles in conjunction with leg and arm movements. Each exercises was repeated 10 times and increased in difficulty until the subject could not maintain the registered air pressure at $40 \pm 10 \mathrm{mmHg}$.

In the placebo exercise intervention, the participants performed automated cycling exercise on an automatic bicycle (Reck Motomed Viva; RECK Technik, Germany). The participants were relaxed in a supine crook-lying position on the Pilates Power Gym Transformer while their legs were attached to the pedals of the automatic bicycle. Passive alternate legs move- 


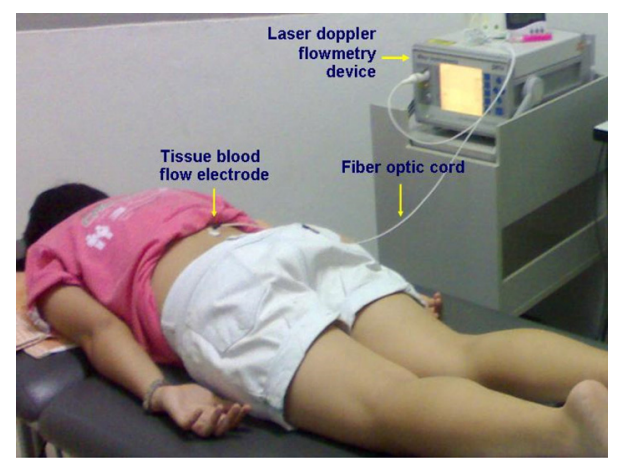

Fig. 1. Measurement of tissue blood flow using LASER Doppler flowmeter device

ments were induced by the automatic bicycle at a speed of 30 revolutions per minute ${ }^{11)}$.

In the control intervention, the participants laid down in a supine crook-lying on the Pilates device but performed no exercises. The participants remained in this position for 15 minutes while both knees were maintained at $70^{\circ}$ of hip flexion and $90^{\circ}$ of knee flexion with the support of a pillow. An experienced musculoskeletal physiotherapist applied the exercise interventions and randomized the order of the application of the treatment, placebo, and control conditions. Another independent investigator measured the experimental outcome measures and was blinded to the applied exercise conditions in the laboratory. The therapist remained blinded to the results of the outcome measures. The participants' blinding was performed by recruiting participants with no previous experience with core stability exercises, and the real aim of the study was not revealed to the participants. Instead, the participants were informed that the study was to investigate the effects of various positioning on pain threshold. Thus, careful measures were taken to maintain the blinding of the therapists and participants in the study.

One of the main study outcome measure, the measurement of TBF, was performed per a previously established protocol ${ }^{12)}$. A laser Doppler blood flow meter (DRT4; Moor Instruments, UK) was used to measure the TBF in fluxes per minute. The participants laid down in the prone position by placing their arms by the sides of their bodies. The target tissue at the most tender spot on the posterior aspect of the lumbosacral area between first lumbar and fifth sacral vertebrae was identified and marked on the body. The electrode of the laser Doppler blood flow meter was put over the center of the target area on the marked body area (Fig. 1). The TBF was measured for every minute for a period of 5 minutes during pre- and immediately post-experimental conditions. The mean value of the TBF was used for the analysis.

Another outcome measure, lumbopelvic stability, was measured with all of the participants in the supine crook-lying position per an established protocol ${ }^{12)}$. The hip was flexed to $70^{\circ}$ to maintain the lumbar spine in the neutral position. A series of seven LPS tests was performed for each participant based on their lumbopelvic control ability. Lumbopelvic control was monitored using a pressure biofeedback device that was inflated to $40 \mathrm{mmHg}$ and placed underneath between each participant's second and fourth lumbar vertebrae. The participants performed a unilateral leg lift in the sagittal plane as the test movement at the end of exhalation after breathing in and out while holding the abdominal hallowing action throughout the test movement. A successful performance was defined as the ability to maintain the registered pressure at $40 \mathrm{mmHg}$ during the testing movement. The LPS test was stopped when the participants were not able to hold $40 \pm 2 \mathrm{mmHg}$ in the biofeedback device.

A total of 25 participants were included after we calculated the sample size with the G*power program for an $\alpha$ level of 0.05 and power analysis of 0.80 with an estimated effect size of 0.54 . The study data were analyzed using SPSS for Windows version 20.0 (SPSS, USA). Examination of the normality of the data using the Shapiro-Wilk test showed normal TBF and LPS data distributions. The changes in the TBF within and between the groups were analyzed using repeated measures analysis of variance (ANOVA). The percentage change in the TBF and LPS values were measured by calculating the variations between pre- and post-change data divided by 100 .

\section{RESULTS}

A total of seven men and 18 women (mean age, $33.33 \pm 14.37$ years) participated in this study. The average height and weight was $162.40 \pm 10.66 \mathrm{~cm}$ and $58.42 \pm 9.66 \mathrm{~kg}$, respectively. The mean duration of chronic non-specific low back pain reported by the participants was $40.36 \pm 35.55$ months with a reported pain intensity of $4.29 \pm 1.81$ on the pain visual analog scale. There were no significant differences noted in any of the baseline variables in the three exercise conditions ( $p$ $>0.10)$. The repeated measures ANOVA results demonstrated a significant increase in blood flow throughout the tissues of the lumbopelvic region after LPST $(p<0.05)$ and a total increase of almost $54 \%$ in the TBF after LPST $(p<0.001)$. Also, the observed trend of increased blood flow throughout the tissues in the lumbopelvic region was significantly higher than the 
Table 1. Data of tissue blood flow and lumbopelvic stability outcomes and percentage change $(\% \mathrm{Ch})$ values are showed as mean \pm standard deviation (SD).

\begin{tabular}{|c|c|c|c|c|c|c|c|c|c|}
\hline \multirow{3}{*}{ Outcomes } & \multicolumn{9}{|c|}{ Conditions } \\
\hline & \multicolumn{3}{|c|}{ Core exercise } & \multicolumn{3}{|c|}{ Placebo } & \multicolumn{3}{|c|}{ Control } \\
\hline & Pre- & Post- & $\% \mathrm{Ch}$ & Pre- & Post- & $\% \mathrm{Ch}$ & Pre- & Post- & $\% \mathrm{Ch}$ \\
\hline BF (flux/min) & $\begin{array}{c}9.02 \\
(2.81)\end{array}$ & $\begin{array}{l}13.56^{* * *} \\
(5.39)\end{array}$ & $\begin{array}{c}54.84 \% \text { a,b } \\
(58.37)\end{array}$ & $\begin{array}{l}10.34 \\
(4.83)\end{array}$ & $\begin{array}{l}10.19 \\
(3.59)\end{array}$ & $\begin{array}{l}-4.00 \% \\
(22.18)\end{array}$ & $\begin{array}{l}10.41 \\
(3.66)\end{array}$ & $\begin{array}{l}10.10 \\
(3.54)\end{array}$ & $\begin{array}{c}-0.19 \% \\
(19.57)\end{array}$ \\
\hline $\begin{array}{l}\text { LPST (mmHg) } \\
\text { (7 levels) }\end{array}$ & $\begin{array}{c}2.20 \\
(0.41)\end{array}$ & $\begin{array}{l}2.72^{* * *} \\
(0.46)\end{array}$ & $\begin{array}{c}26.00 \% \text { a,b } \\
(25.50)\end{array}$ & $\begin{array}{c}2.24 \\
(0.44)\end{array}$ & $\begin{array}{c}2.24 \\
(0.44)\end{array}$ & $\begin{array}{l}0.0 \% \\
(0.00)\end{array}$ & $\begin{array}{c}2.24 \\
(0.44)\end{array}$ & $\begin{array}{c}2.24 \\
(0.44)\end{array}$ & $\begin{array}{l}0.0 \% \\
(0.00)\end{array}$ \\
\hline
\end{tabular}

BF: blood flow; LPST: lumbopelvic core stabilization test

No significant differences in baseline data among the three conditions $(p \geq 0.10)$

${ }^{a}$ Significant differences between placebo $(\mathrm{p}<0.05)$

${ }^{b}$ Significant differences between control $(\mathrm{p}<0.05)$

Significant differences between pre-post $(* \mathrm{p}<0.05 ; * * \mathrm{p}<0.01 ; * * * \mathrm{p}<0.001)$

placebo $(\mathrm{p}<0.05)$ and control intervention $(\mathrm{p}<0.05)$. A similar pattern of a significant increase in LPS level after LPST was also noted $(p<0.001)$, while the increase in LPS was significantly higher in the LPST group than in the placebo and control interventions $(\mathrm{p}<0.05)$. Table 1 shows the mean TBF values and LPS changes in the three exercise intervention groups.

\section{DISCUSSION}

The findings of this study demonstrated that a single LPST session could induce physiological changes by improving TBF by approximately $54 \%$, a value that was significantly greater than that of the placebo and control conditions. Increased TBF to the pathological area may help facilitate the healing process by supplying more oxygen, nutrients, and hormones to the affected area as well as removing waste products and irritant substances from the sensitive tissues. LPST also provided specific core stability in the lumbopelvic region. Thus, the current study observed an increase in the circulatory and mechanical effects as evidenced by significant improvements in the TBF and LPS levels among patients with chronic non-specific low back pain. Previous studies suggested that LPST improves motor control within the lumbopelvic region and can induce inter-segmental stiffness, prevent shear force that causes injury to the lumbar spine, and alleviate pain among patients with chronic low back pain ${ }^{1-7)}$. The current study added knowledge to the above notion that increased TBF may be one clinical reason behind the improvement in pain symptoms and LPS levels among patients with chronic low back pain. Two important theories, namely the vicious cycle theory and a new pain adaptation theory, may support such interactive clinical reasoning behind the observed circulatory-mechanical effects of LPST ${ }^{20,22)}$.

The vicious cycle theory proposes thoughts for clinical reasoning regarding the ischemic spasm induced in the muscles of the lumbopelvic region due to vascular compromise ${ }^{20)}$. The ischemic spasm might contribute to the accumulation of pain substances in the tissues that may cause pain within the lumbopelvic region. As per the vicious cycle theory, pain may trigger the excitability of gamma motor neurons and lead to the development of increased muscle tension or spasm ${ }^{22)}$. In turn, the pain may further prevent free movements within the lumbopelvic region, leading to further spasm-pain-spasm cycles. On the other hand, the pain adaptation theory suggests that pain reduces lumbopelvic muscle contractility, thereby making them work inefficiently. Such inefficiency may reflected by several dysfunction of the lumbopelvic region, including a reduced ability to activate the agonist muscles, time delay in the feed-forward mechanism, and a reduced maximum output from the agonist muscle ${ }^{22}$. This may eventually lead to poor function of the lumbopelvic muscles and poor lumbopelvic stability. One of the interesting findings in the current study is that both LPS and TBF increase after LPST. Exercise training reportedly increases skeletal muscle oxygenation by increasing the capillary network, improves adenosine triphosphate production, and decreases lactic acid production in the muscles and blood ${ }^{23}$. Therefore, improved TBF over the lumbopelvic muscles may have resulted in improved low back pain condition and LPS and vice versa. Furthermore, the findings substantiated the role of a circulatory mechanism behind LPS improvement as an additional reason to those such as improved proprioception, promotion of the tissue healing process, dorsal horn inhibition, and descending pain control inhibition ${ }^{24)}$. Nevertheless, the above stated vascular and mechanical changes did not occur in the placebo and control group since those participants did not perform any voluntary exercises of the lumbopelvic muscles.

One of the important factors that modulates nociceptor sensitivity is the acidity $(\mathrm{pH})$ of the surrounding tissue in the lumbopelvic region ${ }^{25}$ ). An altered $\mathrm{pH}$ of the local muscles of the lumbopelvic region is related to the accumulation of inflammatory soup and mechanical sensitization of the peripheral and central nociceptors among patients with chronic non-specific low back pain ${ }^{26,27)}$. Therefore, the goal of rehabilitation is to increase circulation and movements to the lumbopelvic region to wash out the retained inflammatory mediators and facilitate muscle activation. In such instances, exercise interventions such as motor control and movement strategy training were recommended to influence muscle activation of the lumbopelvic region $^{20)}$. The similar above stated principle could be applicable to the current study since LPST improved movements that 
in turn improved TBF, which might reduce inflammatory mediators and stressors and subsequently improve the movement pattern and LPS in patients with chronic non-specific low back pain. To prove this theory, future studies should investigate the circulating inflammatory marker serotonin and stress marker cortisol after LPST in patients with chronic non-specific low back pain.

The current study may have some limitations. First, it investigated only the immediate effects of LPST on TBF and lumbopelvic stability. Second, since it lacked long-term follow-up, the long-term circulatory-mechanical therapeutic effects of LPST cannot be fully understood in patients with chronic non-specific low back pain. However, in the current study, the specific mechanism of LPST on the TBF and LPS was also pointed out by the addition of a placebo exercise intervention and rest intervention as controlled comparisons. Therefore, the current study potentially provides some understanding of the notion of improved TBF as one of the mechanism behind LPS and low back pain improvement. LPST has clinical implications through mechanical and chemical effects. LPST might help reduce muscle spasms, remove waste metabolites, improve blood circulation, and reduce nociceptive stimulants in the back muscles. The mechanical implications of LPST might include reduced irritation of mechanical structures and improved afferent discharge through the gaining of lumbopelvic region motor control.

In conclusion, the current study's findings support an increase in TBF in the lumbopelvic region and improvement in LPS after LPST among patients with chronic non-specific low back pain. Hence, clinicians might consider LPST an additional therapeutic approach to managing lumbopelvic pain and dysfunction.

\section{ACKNOWLEDGEMENTS}

We express our gratitude to all of the study participants as well as the Thailand Research Fund in conjunction with the Ministry of University Affairs (MUA) for supporting the grant for this study (MRG5380072). We also send special thanks to Prof. Dr. Bill Vicenzino and Prof. Dr. Watchara Kasinrerk for their valuable guidance.

\section{REFERENCES}

1) Hodges PW, Richardson CA: Inefficient muscular stabilization of the lumbar spine associated with low back pain. A motor control evaluation of transversus abdominis. Spine, 1996, 21: 2640-2650. [Medline] [CrossRef]

2) Hides JA, Jull GA, Richardson CA: Long-term effects of specific stabilizing exercises for first-episode low back pain. Spine, 2001, 26: E243-E248. [Medline] [CrossRef]

3) Panjabi MM: The stabilizing system of the spine. Part I. Function, dysfunction, adaptation, and enhancement. J Spinal Disord, 1992, 5: 383-389, discussion 397. [Medline] [CrossRef]

4) Hodges PW, Moseley GL: Pain and motor control of the lumbopelvic region: effect and possible mechanisms. J Electromyogr Kinesiol, 2003, 13: 361-370. [Medline] [CrossRef]

5) Yoo YD, Lee YS: The effect of core stabilization exercises using a sling on pain and muscle strength of patients with chronic low back pain. J Phys Ther Sci, 2012, 24: 671-674. [CrossRef]

6) O'Sullivan PB, Phyty GD, Twomey LT, et al.: Evaluation of specific stabilizing exercise in the treatment of chronic low back pain with radiologic diagnosis of spondylolysis or spondylolisthesis. Spine, 1997, 22: 2959-2967. [Medline] [CrossRef]

7) Ota M, Kaneoka K, Hangai M, et al.: Effectiveness of lumbar stabilization exercises for reducing chronic low back pain and improving quality-of-life. J Phys Ther Sci, 2011, 23: 679-681. [CrossRef]

8) Cairns MC, Foster NE, Wright C: Randomized controlled trial of specific spinal stabilization exercises and conventional physiotherapy for recurrent low back pain. Spine, 2006, 31: E670-E681. [Medline] [CrossRef]

9) Ferreira ML, Ferreira PH, Latimer J, et al.: Comparison of general exercise, motor control exercise and spinal manipulative therapy for chronic low back pain: A randomized trial. Pain, 2007, 131: 31-37. [Medline] [CrossRef]

10) Paungmali A, Hancharoenkul B, Sitilertpisan P, et al.: Hypoalgesic Effects of Sport Massage and Lumbo-Pelvic Core Stabilization on Female Elite National Weightlifters. The Annual British Pain Society Scientific Conference, Manchester, United Kingdom, 13-15 April 2010.

11) Nielsen MM, Mortensen A, Sørensen JK, et al.: Reduction of experimental muscle pain by passive physiological movements. Man Ther, 2009, 14: 101-109. [Medline] [CrossRef]

12) Paungmali A, Sitilertpisan P, Taneyhill K, et al.: Intrarater reliability of pain intensity, tissue blood flow, thermal pain threshold, pressure pain threshold and lumbo-pelvic stability tests in subjects with low back pain. Asian J Sports Med, 


\section{2, 3: 8-14. [Medline] [CrossRef]}

13) Richardson C, Toppenberg R, Jull G: An initial evaluation of eight abdominal exercises for their ability to provide stabilisation for the lumbar spine. Aust J Physiother, 1990, 36: 6-11. [Medline] [CrossRef]

14) Vleeming A, Albert HB, Östgaard HC, et al.: European guidelines for the diagnosis and treatment of pelvic girdle pain. Eur Spine J, 2008, 17: 794-819. [Medline] [CrossRef]

15) Cholewicki J, Panjabi MM, Khachatryan A: Stabilizing function of trunk flexor-extensor muscles around a neutral spine posture. Spine, 1997, 22: 2207-2212. [Medline] [CrossRef]

16) Leonard JH, Paungmali A, Sitilertpisan P, et al.: Changes in transversus abdominis muscle thickness after lumbopelvic core stabilization training among chronic low back pain individuals. Clin Ter, 2015, 166: e312-e316. [Medline]

17) Yang HS, Lee YS, Jin SA: Effect of evidence-based trunk stability exercises on the thickness of the trunk muscles. J Phys Ther Sci, 2015, 27: 473-475. [Medline] [CrossRef]

18) Pool-Goudzwaard AL, Vleeming A, Stoeckart R, et al.: Insufficient lumbopelvic stability: a clinical, anatomical and biomechanical approach to 'a-specific' low back pain. Man Ther, 1998, 3: 12-20. [Medline] [CrossRef]

19) Snijders CJ, Vleeming A, Stoeckart R: Transfer of lumbosacral load to iliac bones and legs Part 1: biomechanics of self-bracing of the sacroiliac joints and its significance for treatment and exercise. Clin Biomech (Bristol, Avon), 1993, 8: 285-294. [Medline] [CrossRef]

20) Hodges PW: Pain and motor control: from the laboratory to rehabilitation. J Electromyogr Kinesiol, 2011, 21: 220-228. [Medline] [CrossRef]

21) Mani R, Cooper C, Kidd BL, et al.: Use of laser Doppler flowmetry and transcutaneous oxygen tension electrodes to assess local autonomic dysfunction in patients with frozen shoulder. J R Soc Med, 1989, 82: 536-538. [Medline]

22) Wright A: Recent concepts in the neurophysiology of pain. Man Ther, 1999, 4: 196-202. [Medline] [CrossRef]

23) UchiyamaK, Miaki H, Terada S, et al.: Effect of muscle strength training and muscle endurance training on muscle deoxygenation level and endurance performance. J Phys Ther Sci, 2011, 23: 349-355. [CrossRef]

24) Yu X, Wang X, Zhang J, et al.: Changes in pressure pain thresholds and Basal electromyographic activity after instrument-assisted spinal manipulative therapy in asymptomatic participants: a randomized, controlled trial. J Manipulative Physiol Ther, 2012, 35: 437-445. [Medline] [CrossRef]

25) Dray A: Inflammatory mediators of pain. Br J Anaesth, 1995, 75: 125-131. [Medline] [CrossRef]

26) Handwerker HO, Reeh PW: Nociceptors, chemosensitivity and sensitization by chemical agents. In: Hyperalgesia and Allodynia, WD Willis (Ed.). New York: Raven Press, 1992, pp 107-115.

27) Zusman M: Mechanisms of musculoskeletal physiotherapy. Phys Ther Rev, 2004, 9: 39-49. [CrossRef] 